\title{
Domestic Terrorism, Investment and Economic Growth: Evidence from Developing Countries
}

\author{
Dr. MUHAMMAD AWAIS ANWAR \\ Assistant Professor, Department of Economics and Business Administration, \\ University of Education. Lahore, Pakistan. \\ Email: awais.anwar@ue.edu.pk \\ Tel: +923324165092 \\ Dr. NOMAN ARSHED \\ Lecturer, Department of Economics, \\ University of Management and Technology. Lahore, Pakistan. \\ Email: noman.arshed@umt.edu.pk \\ Tel: +923364376860

\section{MUHAMMAD IBRAHIM SAEED} \\ (Corresponding Author) \\ Research Associate, Office of Research Innovation and Commercialization, \\ University of Management and Technology. Lahore, Pakistan. \\ Email: ibrahim.saeed@umt.edu.pk \\ Tel: +923340413900
}

\begin{abstract}
The main objective of the study is to empirically examine the relationship between domestic terrorism, investment and economic growth. The study finds the implication of domestic terrorism on investment and growth among 26 Muslim and 14 Christian developing countries. Data regarding the incidence of terrorism are obtained from Global Terrorism Database (2015). While, economic data are obtained from World Development Indicators (WDI, 2015). The data on external and internal conflict have been extracted from Global Conflict Risk Index (GCRI, 2015) for the time period 1990-2015. Ordinary least square (OLS), feasible generalized least squares (FGLS) and system generalized method of moment (SGMM) approaches were applied to ensure robust results with different specification of models by using dummy variable. The value of Dummy variable is 1 , if country is Muslim otherwise 0. For all specifications, it is confirmed that increase in domestic terrorism will decrease the level of investment directly, but the percentage decrease in investment due to terrorism is high among Muslim as compared to Christian developing economies. The results indicate the public policy efforts to mitigate the loss of private investment which can be done initially by public investments to ensure public safety.
\end{abstract}

Keywords: OLS, FGLS, SGMM, Domestic Terrorism, Muslim and Christian Developing Countries.

\section{Introduction}

Terrorism is the use of threat or violence by a group, sub-national group or individual, to gain political, social or religious objectives through fear (Keefer \& Loayza, 2008; Bandyopadhyay et al., 2014). It is mostly the issue of economic gains that causes the eruption of conflicts. Deprivation and inequality places the various groups of society against each other. Terrorist behavior always evolves from conflicts. Terrorist activities and violent behavior cause fear in the community. It creates an environment of uncertainty and risk. Such situation harmfully affects investment and economic activities (Wagner, 2006). 
Over the last 14 years, about 48,000 incidence of terrorism and 107,000 casualties have been recorded around the globe. Most of these terrorist attacks were experienced by the Muslim developing countries (Global Peace Index, 2015). Figure 1 shows that majority of the terrorist attacks were experienced by Iraq, Afghanistan and Pakistan in last two decades. Iraq experienced more than 12,000; Pakistan and Afghanistan experienced more than 6,000 incidences of terrorism from 1990 to 2015. However, Algeria and turkey faced more than 2,000 attacks in the last two decades.

Figure 1: Total Number of Terrorist Attacks across Muslim Developing Countries from 1990 to 2015

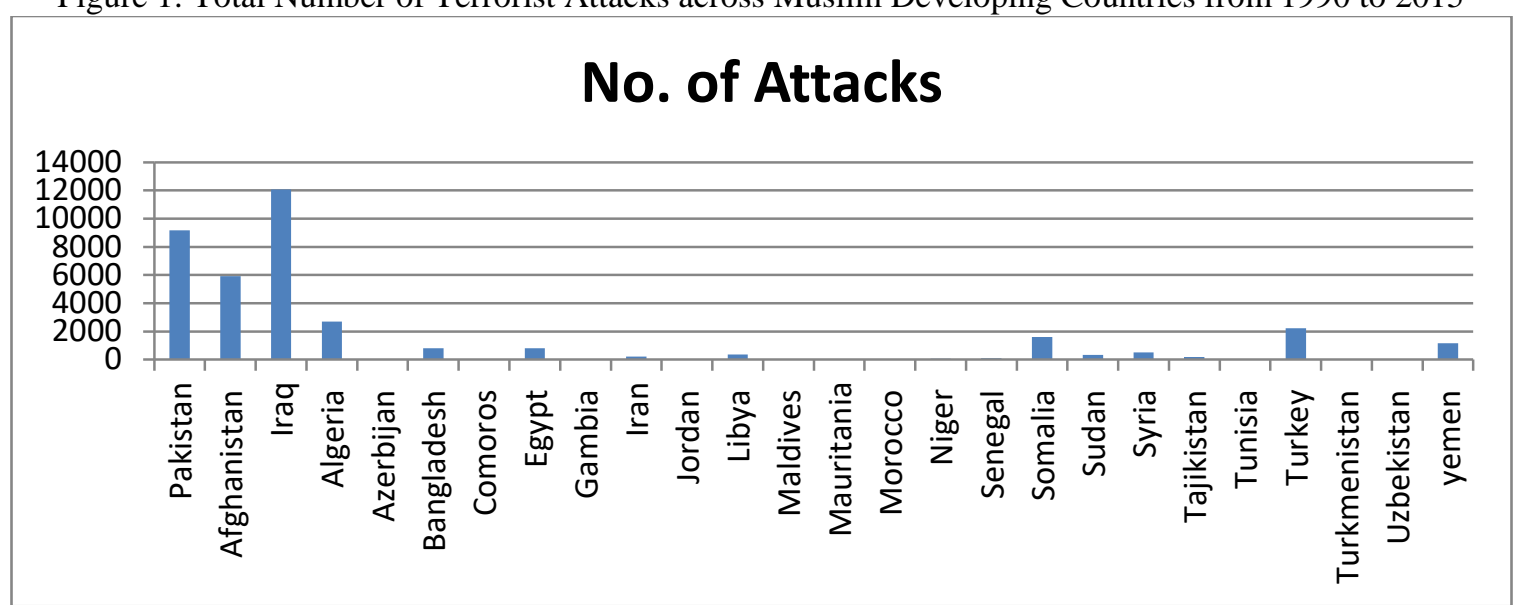

Global Terrorism Database (2015)

Figure 2, presents the situation of terrorism in the Christian developing countries from 1990 to 2015. During this era, majority of the attacks were experienced by Philippine and Peru. Philippine has experienced more than 6,000 attacks from 1990 to 2015. Peru experienced more than 3,000 terrorist attacks during last two decades due to civil conflicts, while, rest of the countries has faced less than 100 attacks from 1990 to 2015. However, incidence of terrorism is very less as compared to Iraq, Afghanistan and Pakistan.

Figure 2: Total Number of Terrorist Attacks across Christian Developing Countries from 1990 to 2015

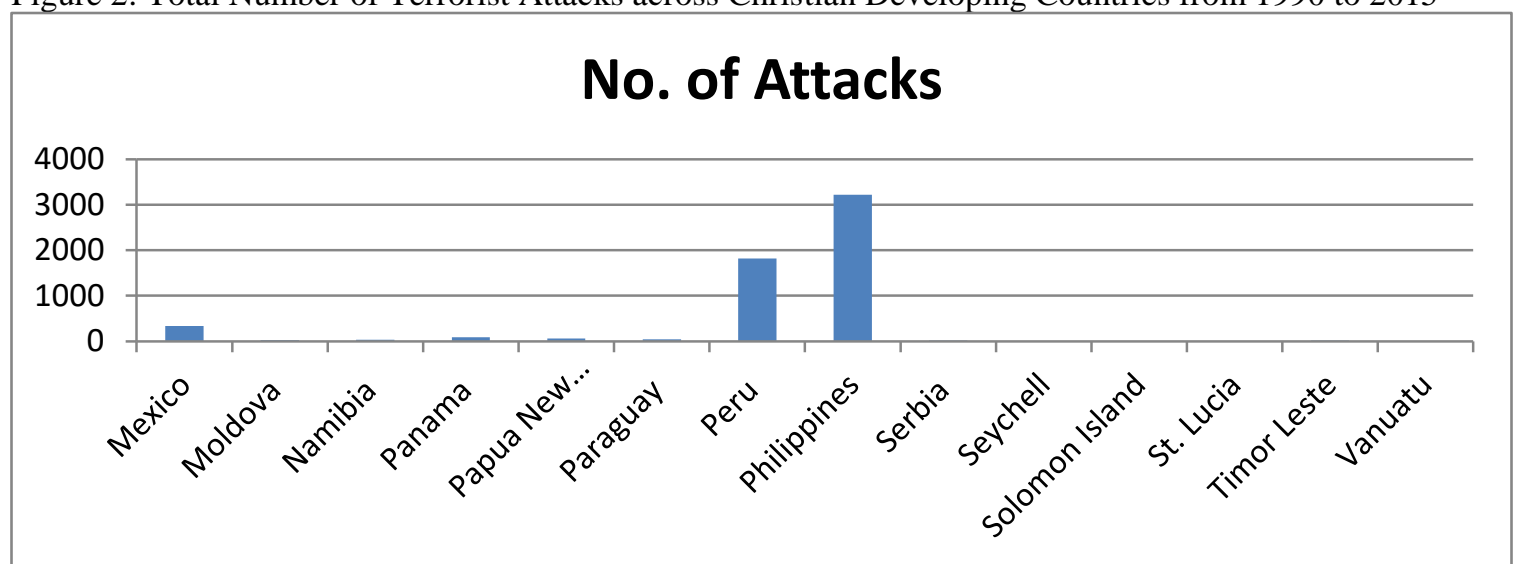

Global Terrorism Database (2015)

Terrorism always comes up with grave economic repercussions as United States of America bore the cost of $\$ 80$ to $\$ 90$ billion after the incidence of $9 / 11$, while, the gross domestic product of USA was $\$ 10$ trillion in 2001 (Kunreuther et al., 2003). The direct cost associated with terrorism comprises of casualties, 
destruction and decline in production, whereas, indirect cost includes an increase in insurance premium and the expenses on security and counter-terrorism measures. Macroeconomic indicators, such as economic growth, government expenditures, investment and consumption also get affected due to terrorism, whereas, rise in terrorism leads to the fall in investment due to higher perceived risk on the part of investors. Such investors try to transfer their funds to safer regions with similar rate of returns (Blomberg et al., 2004).

The study is organized as follows: section II is the literature review Section III incorporates the discussion on data and empirical model with econometric irregularities while section IV explains the estimated results which contribute the deeper understanding that how terrorism effects investment among Muslim's and Christian developing countries. Section V concludes the study with policy implications.

\section{Literature Review}

Becker and Murphy (2001) reveal that high rate of terrorism changes the behavior of individual too. The work of Kalim et al. (2019) argue that the confidence of investor is dependent on the economic condition like terrorist events which compel capital to flight. Their main study objective is to test the asymmetry in effects of terrorism on foreign direct investment. The results of the study reveal that in short run terrorism leads to increase in FDI, later on, it decreases the FDI whereas in long run, the effect of an increase and decrease in terrorism tend to become almost equal and opposite. This phenomenon plays role in deterioration of the economy. As uncertainty and higher cost of borrowing decreases the private and public investment and drive the public expenditure away from productive areas. Both domestic and foreign companies hesitate to invest in a country plaguing with high level of terrorism. Thus, terrorism acts as a cost to investment. Enders et al. (2006) elucidate the association between terrorism and foreign direct investment (FDI) of USA. They use time series and panel data analysis and found that the incidence of 9/11 brings down the FDI of USA. However, in panel estimation of 69 other countries, they found an insignificant relationship between FDI and transnational terrorism.

Moreover, Spich and Grosse (2005) argue that terrorism reduces the cross-national trade and investment when government imposes the anti-terrorism policies. For example, the efficiency of international logistics and shipping will decrease when policies like inspection of shipping containers and security programs are introduced to protect the ports. These findings are consistent with the study of (Czinkota et al., 2004). However, Bouchet (2004) shows that terrorism reduces the risk-taking attitude of managers. The portfolio managers, exporters and lenders feel fear to allocate their assets abroad due to the risk of terrorism. The regions which are characterized by risk and volatility have less FDI and portfolio investment.

Terrorism is found mostly in the areas which are characterized by low economic development, poverty and poor governance. The government of these areas get failed to provide the basic needs, such as infrastructure or the rule of law. On the other hand, foreign direct investment, portfolio investment and cross-country trade reduce the terrorist activity. Sustainability of international business reduces the poverty and other social issues in developing countries. So, the policies which improve the socio-economic situation ultimately reduce the incidence of terrorism ( $\mathrm{Li}$ and Schaub, 2004) ${ }^{1}$. However, Abadie and Gardeazabal (2003) find the relationship between terrorist activity and economic impact on the firms of Spain. They explain that terrorist attacks have an adverse effect on the economic conditions of firm. Furthermore, Glick and Rose (2002) reveal the negative relationship between trade and the number of terrorist attacks. They include 217 countries and the terrorist activities from 1948 to 1997 in sample, whereas, Enders and Hoover (2012) unfold the nonlinear relationship between income and terrorism. They ascertain that GDP per capita has a strong nonlinear effect on transnational terrorism for the sample of 172 countries. Arshed et al. (2019) confirmed that increase in the incidence of victims from terrorism, reduces the investment to register new businesses in the Asian economies.

\footnotetext{
${ }^{1}$ Consistent with the study of (Adler, 2006; Innes, 2007)
} 
The purpose of study is to present the dynamic panel investigation of the overall effect of domestic terrorism and counter terrorism efforts on investment among Muslim and Christian developing economies. It also tries to find the relationship between investment and military expenditures which are used as efforts against terrorism. It aims to make the comparison between the Muslim and Christian developing countries for the effect of terrorism on investment. As the major victims of terrorism are the Muslims; so there is need to check how their investment pattern gets affected by terrorism. There is also a need to compare these investment dynamics with the rest of the developing countries in which majority have the religion, Christianity. The research on terrorism and investment among developing economies having a different religious condition does not gain much attention. Some studies like (Asiedu, 2002; Enders and Sandler, 2006) describe the relationship between FDI and terrorism in developed and developing regions but there are only a few studies which incorporate investment instead of FDI. These studies report the overall impact of Terrorism on FDI in developing or developed economies. They did not separate the Muslim countries; which accounts for the major incidence of terrorism. The separation of countries according to the group of religion is also important because the major victims of terrorism are Muslims.

\section{Methodology, Data and Description of Variables}

The study uses Ordinary Least Square, Feasible Generalized Least Square and System Generalized Method of Moments for the analysis purpose. Two groups comprising of 26 Muslim developing countries and 14 Christian developing countries are taken. Data regarding the incidence of terrorism are obtained from Global Terrorism Database (2015). While, economic data are obtained from World Development Indicators (WDI, 2015). The data on external and internal conflict have been extracted from Global Conflict Risk Index (GCRI, 2015). The dynamic panel data model is used in order to analyze the effect of terrorism on investment of Muslim and Christian developing countries for the period of 1990-2015.

$$
I_{i t}=\alpha+\beta T_{i t}+\varphi_{2} Y_{i t}+\varphi_{3} E_{i t}+\varphi_{4} E_{i t}+\underset{\varepsilon_{i t}}{\varphi_{5} M_{i t}}+\underset{(1)}{\varphi_{6} T_{i t}}+\varphi_{7} I_{i t}+\varphi_{8} E_{i t}+\varphi_{9} I_{i t}+\varphi_{10} D_{i t}+
$$

In the above-mentioned model, $i$ represent the country and $t$ refers the time period. "I" is Investment which is expressed as a percentage of GDP; "T" represents domestic terrorism. Increase in incidence of terrorism leads to decrease the level of investment through fear, risk and uncertainty, (Enders et al., 2006). The study incorporates domestic terrorism, which is calculated as a number of incidents of domestic terrorism annually per 100,000 populations. " $\mathrm{M}$ " is military expenditure as a proxy of counterterrorism efforts because increase in military expenditure will decrease the incidence of terrorism and lead to increase in the level of investment ${ }^{2}$. The dependent variable is investment as percentage of GDP, which represents the change in capital stock. However, "D" represents the dummy variable whose value is "1" for Muslim developing countries; otherwise " 0 ".

Keeping in view the existing review of literature, we draw control variables represented in above equation. "E" is education which exhibits mix results with FDI because decrease in level of education leads to decrease in wage rate and that's why multinational firms wants to operate in a country having lower literacy rate. On contrary, multinational firms need more skilled labor and want to operate in a country having higher literacy rate (Blonigen, 2005). "TO" is Trade openness (sum of imports and exports divided by GDP). Multinational firms want to invest more in open economies because of fewer trade barriers (Busse and Hefeker, 2007). "Y", "In", "Ex" are Economic Growth (Growth of GDP), Inflation (Consumer Price Index) and Exchange rate which measures the expected returns on investment and macroeconomic stability in a country (Bandyopadhyay et al., 2014). "IC" and "EC" are Internal and External Conflict. Higher level of internal and external conflict will decrease the level of investment due to risk of security, (Global report, 2009). An index represents the internal and external conflicts varies from 0-10. If the intensity level of conflict is 0 ; it represents the absence of risk in that country. The risk level from 1-4 covers the conflict, which is conducted without the use of force, while the involvement of force or several

${ }^{2}$ Confirmed by Global Peace Index (2015) 
conflict actors covers the intensity of scale from 5-7 and it also considered as violent conflict. The scale from 8-10 considered as highly violent conflict depending upon the number of causalities, refugees, the personnel involved as well as the other means and consequences.

\section{Estimated Results}

\section{Domestic Terrorism among Muslim and Christian Developing Countries}

In this section, we make comparison among Muslim and Christian Developing countries for the relationship between investment, domestic terrorism and other controlled variables, such as economic growth, trade, education, military expenditure, exchange rate, internal and external conflicts. We divide the countries in two categories that are Muslim and Christian developing Countries.

The value of dummy variable is 1 , if country is Muslim otherwise 0 , which shows that Muslim country is our benchmark category. Initially, study incorporates ordinary least square (OLS) in order to check the sign of variables. Table 1, reveals that all economic variables confirms positive and significant relationship with investment in all specification of model, while domestic terrorism shows negative but insignificant relationship with investment in Table 1 of column (5) and column (4). The results of Table 1 reveal that dummy variable is statistically negative and significant, reflecting that Christian countries have higher level of investment if all variables are independent. The impact of economic growth on investment is greater among Muslim developing countries as compare to Christian developing countries in baseline and fully specified model of column (4) and (5) in Table 1. The unexpected insignificant relationship between domestic terrorism and investment occur due to presence of heteroskedascity and autocorrelation in our model of ordinary least square (OLS).

Table 1: Ordinary Least Square

\begin{tabular}{|c|c|c|c|c|c|}
\hline $\begin{array}{l}\text { Independent } \\
\text { Variables }\end{array}$ & $\begin{array}{l}(1) \\
\text { Base } \\
\text { OLS }\end{array}$ & $\begin{array}{c}(2) \\
\& \text { Internal } \\
\text { Conflict } \\
\text { OLS }\end{array}$ & $\begin{array}{l}(3) \\
\& \text { External } \\
\text { Conflict } \\
\text { OLS }\end{array}$ & $\begin{array}{l}(4) \\
\& \text { Terrorism, } \\
\text { OLS }\end{array}$ & $\begin{array}{c}\text { (5) } \\
\& \text { Terrorism, } \\
\text { Internal conflict, } \\
\text { External conflict } \\
\text { OLS }\end{array}$ \\
\hline GDP growth rate & $\begin{array}{c}7.449 * * * \\
(0.00)\end{array}$ & $\begin{array}{c}7.454 * * * \\
(0.00)\end{array}$ & $\begin{array}{c}7.392 * * * \\
(0.00)\end{array}$ & $\begin{array}{c}7.197 * * * \\
(0.00)\end{array}$ & $\begin{array}{c}6.651 * * * \\
(0.00)\end{array}$ \\
\hline Trade/GDP & $\begin{array}{c}-0.360 * * * \\
(0.00)\end{array}$ & $\begin{array}{c}-0.306 * * * \\
(0.00) \\
\end{array}$ & $\begin{array}{c}-0.366^{* * * *} \\
(0.00)\end{array}$ & $\begin{array}{c}0.548 * * * \\
(0.00)\end{array}$ & $\begin{array}{c}1.677 * * * \\
(0.00)\end{array}$ \\
\hline $\begin{array}{c}\text { Military } \\
\text { Expenditure }\end{array}$ & $\begin{array}{c}1.112 * * * \\
(0.00)\end{array}$ & $\begin{array}{c}1.114 * * * \\
(0.00)\end{array}$ & $\begin{array}{c}1.104 * * * \\
(0.00)\end{array}$ & $\begin{array}{c}1.458 * * * \\
(0.00)\end{array}$ & $\begin{array}{c}1.440 * * * \\
(0.00)\end{array}$ \\
\hline $\begin{array}{c}\text { Ln, Education } \\
\text { (Secondary School } \\
\text { Enrollment) }\end{array}$ & $\begin{array}{l}0.237 \\
(0.77)\end{array}$ & $\begin{array}{l}-0.311 \\
(0.97)\end{array}$ & $\begin{array}{l}0.027 \\
(0.75)\end{array}$ & $\begin{array}{l}0.121 \\
(0.34)\end{array}$ & $\begin{array}{l}0.158 \\
(0.23)\end{array}$ \\
\hline Ln, Exchange rate & $\begin{array}{c}11.529 * * * \\
(0.00)\end{array}$ & $\begin{array}{c}11.775^{* * * *} \\
(0.00) \\
\end{array}$ & $\begin{array}{c}11.4432 * * * \\
(0.00)\end{array}$ & $\begin{array}{c}10.814 * * * \\
(0.00) \\
\end{array}$ & $\begin{array}{c}10.106^{* * * *} \\
(0.00) \\
\end{array}$ \\
\hline$L n$, Inflation & $\begin{array}{c}-6.473 * * * \\
(0.00)\end{array}$ & $\begin{array}{c}-6.664 * * * \\
(0.00)\end{array}$ & $\begin{array}{c}-6.442 * * * \\
(0.00)\end{array}$ & $\begin{array}{c}-5.639 * * * \\
(0.00)\end{array}$ & $\begin{array}{c}-5.101 * * * \\
(0.00)\end{array}$ \\
\hline Internal Conflict & & $\begin{array}{l}0.023 \\
(0.28) \\
\end{array}$ & & & $\begin{array}{c}-0.102 \\
(0.23) \\
\end{array}$ \\
\hline External Conflict & & & $\begin{array}{l}0.032 \\
(0.84) \\
\end{array}$ & & $\begin{array}{l}0.024 \\
(0.36) \\
\end{array}$ \\
\hline $\begin{array}{c}\text { Domestic } \\
\text { Terrorism } \\
\text { (Per 100,000 } \\
\text { persons) } \\
\end{array}$ & & & & $\begin{array}{l}-0.039 \\
(0.93)\end{array}$ & $\begin{array}{l}0.064 \\
(0.88)\end{array}$ \\
\hline
\end{tabular}




\begin{tabular}{|c|c|c|c|c|c|}
\hline GDP growth*D & $\begin{array}{c}6.558 * * * \\
(0.00)\end{array}$ & $\begin{array}{c}6.600 * * * \\
(0.00)\end{array}$ & $\begin{array}{c}6.442 * * * \\
(0.00)\end{array}$ & $\begin{array}{c}6.624 * * * \\
(0.00)\end{array}$ & $\begin{array}{c}6.112 * * * \\
(0.00)\end{array}$ \\
\hline Trade/GDP*D & $\begin{array}{c}0.844 * * * \\
(0.00)\end{array}$ & $\begin{array}{c}0.883 * * * \\
(0.00)\end{array}$ & $\begin{array}{c}0.851 * * * \\
(0.00)\end{array}$ & $\begin{array}{c}1.120 * * * \\
(0.00)\end{array}$ & $\begin{array}{c}-1.196 * * * \\
(0.00)\end{array}$ \\
\hline $\begin{array}{c}\text { Military } \\
\text { Expenditure }\end{array}$ & $\begin{array}{c}0.872 * * * \\
(0.00) \\
\end{array}$ & $\begin{array}{c}0.882^{* * * *} \\
(0.00)\end{array}$ & $\begin{array}{c}0.865^{* * * *} \\
(0.00)\end{array}$ & $\begin{array}{c}1.118 * * * \\
(0.00)\end{array}$ & $\begin{array}{c}1.094 * * * \\
(0.00)\end{array}$ \\
\hline $\begin{array}{c}\text { Ln, Education } \\
\text { (Secondary School } \\
\text { Enrollment)*D }\end{array}$ & $\begin{array}{c}0.823^{* * * *} \\
(0.00)\end{array}$ & $\begin{array}{c}0.846^{* * * *} \\
(0.00)\end{array}$ & $\begin{array}{c}0.822 * * * \\
(0.00)\end{array}$ & $\begin{array}{c}0.557 * * * \\
(0.00)\end{array}$ & $\begin{array}{c}0.538 * * * \\
(0.00)\end{array}$ \\
\hline $\begin{array}{l}\text { Ln, Exchange } \\
\text { rate*D }\end{array}$ & $\begin{array}{c}- \\
10.133 * * * \\
(0.00)\end{array}$ & $\begin{array}{c}-10.337 * * * \\
(0.00)\end{array}$ & $\begin{array}{c}-10.047 * * * \\
(0.00)\end{array}$ & $\begin{array}{c}-7.929 * * * \\
(0.00)\end{array}$ & $\begin{array}{c}-7.254 * * * \\
(0.00)\end{array}$ \\
\hline Ln, Inflation*D & $\begin{array}{c}6.085 * * * \\
(0.00)\end{array}$ & $\begin{array}{c}6.243^{* * * *} \\
(0.00)\end{array}$ & $\begin{array}{c}6.055^{* * * *} \\
(0.00)\end{array}$ & $\begin{array}{c}5.123 * * * \\
(0.00)\end{array}$ & $\begin{array}{c}4.612 * * * \\
(0.00)\end{array}$ \\
\hline $\begin{array}{c}\text { Internal } \\
\text { Conflict*D }\end{array}$ & & $\begin{array}{l}0.012 \\
(0.60)\end{array}$ & & & $\begin{array}{c}-0.050 * * * \\
(0.04)\end{array}$ \\
\hline $\begin{array}{c}\text { External } \\
\text { Conflict*D }\end{array}$ & & & $\begin{array}{l}-0.044 \\
(0.84) \\
\end{array}$ & & $\begin{array}{l}0.024 \\
(0.35) \\
\end{array}$ \\
\hline $\begin{array}{c}\text { Domestic } \\
\text { Terrorism } \\
\text { (Per 100,000 } \\
\text { persons)*D }\end{array}$ & & & & $\begin{array}{l}-0.010 \\
(0.83)\end{array}$ & $\begin{array}{l}-0.053 \\
(0.36)\end{array}$ \\
\hline Dummy & $\begin{array}{c}- \\
17.843 * * * \\
(0.00)\end{array}$ & $\begin{array}{c}-18.625^{* * * *} \\
(0.00)\end{array}$ & $\begin{array}{c}-17.837 * * * \\
(0.00)\end{array}$ & $\begin{array}{c}-9.800 * * * \\
(0.04)\end{array}$ & $\begin{array}{c}-8.524 * * \\
(0.08)\end{array}$ \\
\hline Intercept & $\begin{array}{c}24.432 * * * \\
(0.00)\end{array}$ & $\begin{array}{c}25.178 * * * \\
(0.00)\end{array}$ & $\begin{array}{c}24.406 * * * \\
(0.00)\end{array}$ & $\begin{array}{c}18.672 * * * \\
(0.00)\end{array}$ & $\begin{array}{c}17.024 * * * \\
(0.00)\end{array}$ \\
\hline $\mathrm{R}^{2}$ & 0.84 & 0.85 & 0.85 & 0.86 & 0.86 \\
\hline
\end{tabular}

$\mathrm{P}$ values are presented in round brackets. $* * *$ and $* * *$ represent statistical significance at the $0.10,0.05$ and 0.01 levels, respectively. Models (1)-(5) are different specifications of cross country investmentgrowth regressions. Models (1)-(4) are the basic OLS model adding the different forms of conflict, i.e. terrorism, internal conflict, external conflict and their sum separately

The presence of above discussed problems, shifts our analysis towards feasible generalized least square (FGLS), which resolves the problem of heteroskedascity and autocorrelation. Keeping in view, the fully specified and baseline model in column (4) and (5) of Table 2, reveals the negative and significant relationship between investment and domestic terrorism per 100,000 population, while all other economic variables exhibits positive and significant relationship with investment except trade openness.

Table 2 reveals that dummy variable is positive and significant which shows that level of investment is higher among Muslim developing countries, independent of all variables. This explains that if we assume all factors constant Muslim developing countries have higher level of investment as compare to Christian developing countries. The above discussed results are consistent with the findings of (Shahbaz and Shabir, 2012; Choi, 2014).

In addition, comparison of slope coefficients for the relationship of domestic terrorism per 100,000 population and investment among Muslim and Christian developing countries reveal that percentage decrease in investment due to increase in domestic terrorism per 100,000 population is greater among Muslim developing countries as compare to Christian developing countries in baseline and fully specified model. However, the rest of economic variables show positive and significant relationship with investment except trade openness in column (1), (2) and (3) of Table 2. 
Table 2: Feasible Generalized Least Square

\begin{tabular}{|c|c|c|c|c|c|}
\hline $\begin{array}{c}\text { Independent } \\
\text { Variables }\end{array}$ & $\begin{array}{c}\text { (1) } \\
\text { Base } \\
\text { FGLS }\end{array}$ & $\begin{array}{c}(2) \\
\& \text { Internal } \\
\text { Conflict } \\
\text { FGLS }\end{array}$ & $\begin{array}{c}(3) \\
\& \text { External } \\
\text { Conflict } \\
\text { FGLS }\end{array}$ & $\begin{array}{l}(4) \\
\& \text { Terrorism, } \\
\text { FGLS }\end{array}$ & $\begin{array}{c}\text { (5) } \\
\& \text { Terrorism, } \\
\text { Internal conflict, } \\
\text { External conflict } \\
\text { FGLS }\end{array}$ \\
\hline $\begin{array}{l}\text { GDP growth } \\
\text { rate }\end{array}$ & $\begin{array}{c}0.824 * * * \\
(0.00)\end{array}$ & $\begin{array}{c}0.660 * * * \\
(0.01)\end{array}$ & $\begin{array}{c}1.020 * * * \\
(0.00)\end{array}$ & $\begin{array}{c}0.177 * * * \\
(0.00)\end{array}$ & $\begin{array}{c}1.005^{* * * *} \\
(0.00)\end{array}$ \\
\hline Trade/GDP & $\begin{array}{c}-0.547 * * * \\
(0.00)\end{array}$ & $\begin{array}{c}-0.474 * * * \\
(0.00)\end{array}$ & $\begin{array}{c}-0.224 * * * \\
(0.04)\end{array}$ & $\begin{array}{c}0.487 * * * \\
(0.00)\end{array}$ & $\begin{array}{c}0.848 * * * \\
(0.00)\end{array}$ \\
\hline $\begin{array}{c}\text { Military } \\
\text { Expenditure }\end{array}$ & $\begin{array}{l}0.049 \\
(0.14) \\
\end{array}$ & $\begin{array}{l}0.068 \\
(0.36) \\
\end{array}$ & $\begin{array}{l}0.045 \\
(0.91) \\
\end{array}$ & $\begin{array}{c}0.283 * * * \\
(0.00)\end{array}$ & $\begin{array}{c}0.872 * * * \\
(0.00)\end{array}$ \\
\hline $\begin{array}{c}\text { Ln, Education } \\
\text { (Secondary } \\
\text { School } \\
\text { Enrollment) }\end{array}$ & $\begin{array}{l}0.716 * * * \\
(0.00)\end{array}$ & $\begin{array}{l}0.626 * * * \\
(0.00)\end{array}$ & $\begin{array}{c}0.847 * * * \\
(0.00)\end{array}$ & $\begin{array}{c}0.663 * * * \\
(0.00)\end{array}$ & $\begin{array}{l}0.204 * * * \\
(0.00)\end{array}$ \\
\hline $\begin{array}{l}\text { Ln, Exchange } \\
\text { rate }\end{array}$ & $\begin{array}{c}4.064 * * * \\
(0.00)\end{array}$ & $\begin{array}{l}4.711 * * * \\
(0.00)\end{array}$ & $\begin{array}{c}4.730 * * * \\
(0.00)\end{array}$ & $\begin{array}{c}3.749 * * * \\
(0.00)\end{array}$ & $\begin{array}{c}3.622 * * * \\
(0.00)\end{array}$ \\
\hline Ln, Inflation & $\begin{array}{c}1.873 * * * \\
(0.00)\end{array}$ & $\begin{array}{c}1.929 * * * \\
(0.00)\end{array}$ & $\begin{array}{c}1.162 * * * \\
(0.00)\end{array}$ & $\begin{array}{c}1.801 * * * \\
(0.00)\end{array}$ & $\begin{array}{c}2.284 * * * \\
(0.00)\end{array}$ \\
\hline $\begin{array}{l}\text { Internal } \\
\text { Conflict }\end{array}$ & & $\begin{array}{l}0.024 \\
(0.35)\end{array}$ & & & $\begin{array}{l}0.045 \\
(0.35)\end{array}$ \\
\hline $\begin{array}{l}\text { External } \\
\text { Conflict }\end{array}$ & & & $\begin{array}{c}-0.020 * * * \\
(0.00)\end{array}$ & & $\begin{array}{c}-0.027 * * * \\
(0.00)\end{array}$ \\
\hline $\begin{array}{c}\text { Domestic } \\
\text { Terrorism } \\
\text { (Per 100,000 } \\
\text { persons) } \\
\end{array}$ & & & & $\begin{array}{c}-0.011 * * * \\
(0.00)\end{array}$ & $\begin{array}{c}-0.056^{* * * *} \\
(0.00)\end{array}$ \\
\hline GDP growth*D & $\begin{array}{c}0.353 * * * \\
(0.00)\end{array}$ & $\begin{array}{c}0.143 * * \\
(0.06)\end{array}$ & $\begin{array}{c}0.031 * * * \\
(0.00)\end{array}$ & $\begin{array}{l}0.111 * * * \\
(0.00)\end{array}$ & $\begin{array}{c}0.222 * * * \\
(0.00)\end{array}$ \\
\hline Trade/GDP*D & $\begin{array}{c}0.434 * * * \\
(0.00)\end{array}$ & $\begin{array}{c}0.449 * * * \\
(0.00)\end{array}$ & $\begin{array}{c}0.408 * * * \\
(0.00)\end{array}$ & $\begin{array}{c}-0.094 * * * \\
(0.00)\end{array}$ & $\begin{array}{c}-0.381^{* * *} \\
(0.00)\end{array}$ \\
\hline $\begin{array}{c}\text { Military } \\
\text { Expenditure }\end{array}$ & $\begin{array}{c}0.197 * * * \\
(0.00)\end{array}$ & $\begin{array}{c}0.207 * * * \\
(0.00)\end{array}$ & $\begin{array}{c}0.177 * * * \\
(0.00)\end{array}$ & $\begin{array}{c}0.023 * * * \\
(0.00)\end{array}$ & $\begin{array}{c}0.173 * * * \\
(0.00)\end{array}$ \\
\hline $\begin{array}{l}\text { Ln, Education } \\
\text { (Secondary } \\
\text { School } \\
\text { Enrollment)*D }\end{array}$ & $\begin{array}{c}0.612 * * * \\
(0.00)\end{array}$ & $\begin{array}{c}0.368 * * * \\
(0.05)\end{array}$ & $\begin{array}{c}0.316 * * \\
(0.03)\end{array}$ & $\begin{array}{c}0.663 * * * \\
(0.00)\end{array}$ & $\begin{array}{l}0.191 * * * \\
(0.00)\end{array}$ \\
\hline $\begin{array}{c}\text { Ln, Exchange } \\
\text { rate*D }\end{array}$ & $\begin{array}{c}-1.561 * * * \\
(0.00)\end{array}$ & $\begin{array}{c}-2.542 * * * \\
(0.00)\end{array}$ & $\begin{array}{c}-1.135 * * * \\
(0.00)\end{array}$ & $\begin{array}{l}-0.173 \\
(0.90)\end{array}$ & $\begin{array}{l}0.500 * * * \\
(0.00)\end{array}$ \\
\hline Ln, Inflation*D & $\begin{array}{c}-0.432 * * * \\
(0.00)\end{array}$ & $\begin{array}{c}-0.231 * * * \\
(0.00)\end{array}$ & $\begin{array}{c}-0.318 * * * \\
(0.00)\end{array}$ & $\begin{array}{c}-0.217 * * * \\
(0.00)\end{array}$ & $\begin{array}{c}-0.530 * * * \\
(0.00)\end{array}$ \\
\hline $\begin{array}{c}\text { Internal } \\
\text { Conflict*D }\end{array}$ & & $\begin{array}{l}0.034 \\
(0.43) \\
\end{array}$ & & & $\begin{array}{c}-0.045^{* * *} \\
(0.00) \\
\end{array}$ \\
\hline $\begin{array}{c}\text { External } \\
\text { Conflict*D }\end{array}$ & & & $\begin{array}{l}-0.012 \\
(0.63) \\
\end{array}$ & & $\begin{array}{c}-0.010 * * * \\
(0.00)\end{array}$ \\
\hline $\begin{array}{c}\text { Domestic } \\
\text { Terrorism } \\
\text { (Per } 100,000 \\
\text { persons)*D }\end{array}$ & & & & $\begin{array}{c}-0.019 * * * \\
(0.00)\end{array}$ & $\begin{array}{c}-0.140 * * * \\
(0.00)\end{array}$ \\
\hline Dummy & $1.213 * * *$ & $0.989 * * *$ & $1.057 * * *$ & $1.395 * * *$ & $2.318 * * *$ \\
\hline
\end{tabular}




\begin{tabular}{|c|c|c|c|c|c|}
\hline & $(0.00)$ & $(0.02)$ & $(0.00)$ & $(0.00)$ & $(0.00)$ \\
\hline \multirow{2}{*}{ Intercept } & $0.540^{* * *}$ & $0.503^{*}$ & $0.725^{* * *}$ & $0.702^{* * *}$ & $2.145^{* * *}$ \\
& $(0.00)$ & $(0.10)$ & $(0.00)$ & $(0.00)$ & $(0.00)$ \\
\hline $\mathrm{R}^{2}$ & 0.85 & 0.85 & 0.87 & 0.88 & 0.89 \\
\hline
\end{tabular}

$\mathrm{P}$ values are presented in round brackets. $* ; *$ and $* * *$ represent statistical significance at the $0.10,0.05$ and 0.01 levels, respectively. Models (1)-(5) are different specifications of cross country investmentgrowth regressions. Models (1)-(4) are the basic FGLS model adding the different forms of conflict, i.e. terrorism, internal conflict, external conflict and their sum separately.

Domestic terrorism decreases the level of output, which further reduces the investment. This problem can be resolved by government by using counterterrorism efforts. In addition, national income account identity reveals that economic growth relates with investment endogenously. These two major issues create the problem of endogeneity in our model. In endogeneity, two or more variables jointly determined the behavior of model. In order to overcome this problem, we incorporate system generalized method of moments (SGMM), which kept the additional moment conditions.

Table 3: System Generalized Method of Moments

\begin{tabular}{|c|c|c|c|c|c|}
\hline $\begin{array}{l}\text { Independent } \\
\text { Variables }\end{array}$ & $\begin{array}{c}(1) \\
\text { Base } \\
\text { SGMM }\end{array}$ & $\begin{array}{c}(2) \\
\text { \& Internal } \\
\text { Conflict } \\
\text { SGMM }\end{array}$ & $\begin{array}{c}\text { (3) } \\
\text { \& External } \\
\text { Conflict } \\
\text { SGMM }\end{array}$ & $\begin{array}{l}(4) \\
\& \text { Terrorism, } \\
\text { SGMM }\end{array}$ & $\begin{array}{c}5) \\
\& \text { Terrorism, } \\
\text { Internal conflict, } \\
\text { External conflict } \\
\text { SGMM } \\
\end{array}$ \\
\hline GDP growth rate & $\begin{array}{c}1.880 * * * \\
(0.00)\end{array}$ & $\begin{array}{c}0.836^{* * * *} \\
(0.00)\end{array}$ & $\begin{array}{c}2.404 * * * \\
(0.00)\end{array}$ & $\begin{array}{c}0.122 * * * \\
(0.00)\end{array}$ & $\begin{array}{l}0.878 \\
(0.00)\end{array}$ \\
\hline Trade/GDP & $\begin{array}{l}-0.677 \\
(0.468) \\
\end{array}$ & $\begin{array}{c}0.261 * * * \\
(0.00)\end{array}$ & $\begin{array}{c}0.484 * * * \\
(0.00)\end{array}$ & $\begin{array}{c}-0.253 * * * \\
(0.00)\end{array}$ & $\begin{array}{l}0.940 \\
(0.00) \\
\end{array}$ \\
\hline $\begin{array}{c}\text { Military } \\
\text { Expenditure }\end{array}$ & $\begin{array}{c}0.477 * * * \\
(0.00) \\
\end{array}$ & $\begin{array}{c}0.486^{* * * *} \\
(0.00)\end{array}$ & $\begin{array}{c}0.613 * * * \\
(0.00)\end{array}$ & $\begin{array}{c}0.323^{* * * *} \\
(0.00)\end{array}$ & $\begin{array}{l}0.130 \\
(0.00) \\
\end{array}$ \\
\hline $\begin{array}{c}\text { Ln, Education } \\
\text { (Secondary School } \\
\text { Enrollment) }\end{array}$ & $\begin{array}{c}0.758^{* * *} \\
(0.00)\end{array}$ & $\begin{array}{c}0.453^{* * * *} \\
(0.00)\end{array}$ & $\begin{array}{c}0.662 * * * \\
(0.00)\end{array}$ & $\begin{array}{c}0.353^{* * * *} \\
(0.00)\end{array}$ & $\begin{array}{l}0.302 \\
(0.00)\end{array}$ \\
\hline$L n$, Exchange rate & $\begin{array}{c}-0.842 * * * \\
(0.00)\end{array}$ & $\begin{array}{c}0.136^{* * * *} \\
(0.00)\end{array}$ & $\begin{array}{c}2.214 * * * \\
(0.00)\end{array}$ & $\begin{array}{c}4.662 * * * \\
(0.00)\end{array}$ & $\begin{array}{l}4.503 \\
(0.00)\end{array}$ \\
\hline$L n$, Inflation & $\begin{array}{c}1.179 * * * \\
(0.00)\end{array}$ & $\begin{array}{c}2.040 * * * \\
(0.00)\end{array}$ & $\begin{array}{c}0.803 * * * \\
(0.00)\end{array}$ & $\begin{array}{c}1.985 * * * \\
(0.00)\end{array}$ & $\begin{array}{l}2.165 \\
(0.00)\end{array}$ \\
\hline Internal Conflict & & $\begin{array}{c}0.010^{* * * *} \\
(0.00)\end{array}$ & & & $\begin{array}{l}-0.021 \\
(0.11) \\
\end{array}$ \\
\hline External Conflict & & & $\begin{array}{c}-0.014 * * \\
(0.07) \\
\end{array}$ & & $\begin{array}{l}0.012 \\
(0.08) \\
\end{array}$ \\
\hline $\begin{array}{c}\text { Domestic } \\
\text { Terrorism } \\
\text { (Per 100,000 } \\
\text { persons) }\end{array}$ & & & & $\begin{array}{c}-0.195 * * * \\
(0.00)\end{array}$ & $\begin{array}{l}-0.021 \\
(0.00)\end{array}$ \\
\hline GDP growth*D & $\begin{array}{c}0.887 * * * \\
(0.00)\end{array}$ & $\begin{array}{c}0.123 * * * \\
(0.00)\end{array}$ & $\begin{array}{c}0.354 * * * \\
(0.00)\end{array}$ & $\begin{array}{c}0.136^{* * * *} \\
(0.00)\end{array}$ & $\begin{array}{l}0.107 \\
(0.00)\end{array}$ \\
\hline Trade/GDP*D & $\begin{array}{c}0.127 * * * \\
(0.00)\end{array}$ & $\begin{array}{l}0.638 \\
(0.94)\end{array}$ & $\begin{array}{c}0.880 * * * \\
(0.00)\end{array}$ & $\begin{array}{c}0.887 * * * \\
(0.00)\end{array}$ & $\begin{array}{l}0.945 \\
(0.10)\end{array}$ \\
\hline $\begin{array}{c}\text { Military } \\
\text { Expenditure }\end{array}$ & $\begin{array}{c}0.121 * * * \\
(0.00)\end{array}$ & $\begin{array}{c}0.123^{* * * *} \\
(0.00)\end{array}$ & $\begin{array}{c}0.144 * * * \\
(0.00)\end{array}$ & $\begin{array}{c}0.530 * * * \\
(0.00)\end{array}$ & $\begin{array}{l}0.130 \\
(0.00)\end{array}$ \\
\hline
\end{tabular}




\begin{tabular}{|c|c|c|c|c|c|}
\hline $\begin{array}{c}\text { Ln, Education } \\
\text { (Secondary School } \\
\text { Enrollment)*D }\end{array}$ & $\begin{array}{c}0.692 * * * \\
(0.00)\end{array}$ & $\begin{array}{c}0.043 * * * \\
(0.00)\end{array}$ & $\begin{array}{c}0.134 * * * \\
(0.00)\end{array}$ & $\begin{array}{c}0.190 * * * \\
(0.00)\end{array}$ & $\begin{array}{l}0.160 \\
(0.00)\end{array}$ \\
\hline $\begin{array}{c}\text { Ln, Exchange } \\
\text { rate*D }\end{array}$ & $\begin{array}{c}2.244 * * * \\
(0.00)\end{array}$ & $\begin{array}{c}2.431 * * * \\
(0.00)\end{array}$ & $\begin{array}{c}2.476^{* * * *} \\
(0.00)\end{array}$ & $\begin{array}{c}-0.537 * * * \\
(0.00)\end{array}$ & $\begin{array}{l}0.405 \\
(0.00)\end{array}$ \\
\hline Ln, Inflation*D & $\begin{array}{c}-0.293 * * * \\
(0.00)\end{array}$ & $\begin{array}{c}0.364 * * * \\
(0.00)\end{array}$ & $\begin{array}{c}-0.518 * * * \\
(0.00)\end{array}$ & $\begin{array}{c}-0.106 * * * \\
(0.00)\end{array}$ & $\begin{array}{l}-0.507 \\
(0.00)\end{array}$ \\
\hline $\begin{array}{c}\text { Internal } \\
\text { Conflict*D }\end{array}$ & & $\begin{array}{c}0.009 * * * \\
(0.00)\end{array}$ & & & $\begin{array}{l}-0.045 \\
(0.00)\end{array}$ \\
\hline $\begin{array}{c}\text { External } \\
\text { Conflict*D }\end{array}$ & & & $\begin{array}{c}0.013 * * * \\
(0.00)\end{array}$ & & $\begin{array}{l}0.023 \\
(0.00)\end{array}$ \\
\hline $\begin{array}{c}\text { Domestic } \\
\text { Terrorism } \\
\text { (Per 100,000 } \\
\text { persons)*D }\end{array}$ & & & & $\begin{array}{c}-0.051 * * * \\
(0.00)\end{array}$ & $\begin{array}{l}-0.904 \\
(0.00)\end{array}$ \\
\hline Dummy & $\begin{array}{c}-0.170 * * * \\
(0.00)\end{array}$ & $\begin{array}{c}0.043 * * * \\
(0.00)\end{array}$ & $\begin{array}{c}0.761 * * * \\
(0.00)\end{array}$ & $\begin{array}{c}1.002 * * * \\
(0.00)\end{array}$ & $\begin{array}{l}0.268 \\
(0.00)\end{array}$ \\
\hline Intercept & $\begin{array}{c}-0.754 * * * \\
(0.00)\end{array}$ & $\begin{array}{c}0.771 * * * \\
(0.00)\end{array}$ & $\begin{array}{c}0.178 * * * \\
(0.00)\end{array}$ & $\begin{array}{c}0.179 * \\
(0.10)\end{array}$ & $\begin{array}{l}0.338 \\
(0.00)\end{array}$ \\
\hline Sargan Test & 0.02 & 0.04 & 0.46 & 0.26 & 0.36 \\
\hline
\end{tabular}

$\mathrm{P}$ values are presented in round brackets. $* ; *$ and $* * *$ represent statistical significance at the $0.10,0.05$ and 0.01 levels, respectively. Models (1)-(5) are different specifications of cross country investmentgrowth regressions. Models (1)-(4) are the basic SGMM model adding the different forms of conflict, i.e. terrorism, internal conflict, external conflict and their sum separately.

The results of system generalized method of moments (SGMM) reveals that domestic terrorism shows negative and significant relationship with investment in fully specified and baseline model in column (4) and (5) of Table 3. The effect of economic growth and military expenditure on investment is greater among Muslim developing countries as compare to Christian developing countries. The effect of military expenditure on investment is greater among Muslim developing countries is due to the rising rate of incidence of terrorism. The government spends more on the security of country in order to reduce the terrorist attacks. The above discussed results are consistent with the findings of (Abadie and Gardeazabal, 2008; Kunreuther et al., 2003; Li and Schaub, 2004; Spich and Grosse, 2005; Enders et al., 2006).

However, rest of economic variables such as economic growth, trade openness, education, inflation, military expenditure and exchange rate exhibits positive and significant relationship with investment, while internal conflict shows negative and significant relationship with investment. The positive and significant dummy variable in Table 3 shows that Muslim developing countries have high level of investment; independent of all variables. However, the percentage decrease in investment due to domestic terrorism is greater among Muslim developing countries as compare to Christian developing countries because the number of terrorist incidences is greater among Muslim developing countries.

\section{Discussion}

Our results suggest the negative relationship between terrorism and investment in all specification of model because investor hesitates to invest in a country pertaining to high level of terrorist attacks due to risk and uncertainty. Increase in incidence of terrorism force government to invest in non-development expenditures such as security of people and their investment to curtail the level of terrorism in a country. The portfolio managers, exporters and lenders feel fear to allocate their assets in abroad because of risk due to terrorism. The areas which are characterized by risk and volatility have less FDI and portfolio investment (Czinkota et al., 2004; Bouchet, 2004; Spich and Grossee, 2005). Considering the example of Pakistan, The pertaining 
war against terrorism has severe repercussion on Pakistan's level of investment because investors of industrial sectors do not have secure access to European markets due to pertaining risk to their shipments (Shahbaz, 2013). Majority of terrorist attacks occur in Muslim countries, that's why decrease in percentage of investment due to incidence of terrorism is greater among Muslim countries as compare to Christian countries.

The Keynesian school of thought believes that increase in military expenditure will lead to increase in demand and purchasing power. In order to meet the demand, the investor will invest more and this will increase the real output (Narayan et al., 2007). Economies pertaining to high level of terrorism such as Pakistan will curtail the problem of terrorism by the use of military. Increase in military expenditure increase the level of investment due to dampening the effect of terrorism in a country. Due to which investor feel secure to invest in a country (Shahbaz, 2013). Military use the idle resources in more effective way in other economic sectors. However, military establishment develops and create new skills which is injected in labor force and develop management and organizational structure, which is useful for economic growth (Deger and Sen, 1983).

Rest of the economic variables exhibits mix results with different specification of model. Education represent the mix results with investment because multinational firms want to prefer operation in countries with low literacy rate, while multinational firms also require high skilled labor force and they choose country with high literacy rate (Blonigen, 2005). Open economies are mostly favored by export oriented investment, (Busse and Hefeker, 2007). The higher intensity of internal and external conflict reduces the level of FDI because of higher security risk (Global report, 2009). Accelerator theory of investment reveals that increase in economic growth will lead to increase in investment in order to meet the future demand the investor will invest more (Blomberg and Hess, 2008).

Increase in inflation leads to decrease in investment because it minimizes the real saving and investment returns. Majority of the investor set their long term objectives for long term purchasing power. Inflation puts this goal at risk because investment returns must first keep up with the rate of inflation in order to increase real purchasing power. For example, an investment that returns $2 \%$ before inflation in an environment of $3 \%$ inflation will actually produce a negative return $(-1 \%)$ when adjusted for inflation. In much the same way, rising inflation erodes the value of the principal on fixed income securities. Suppose an investor buys a five-year bond with a principal value of $\$ 100$. If the rate of inflation is $3 \%$ annually, the value of the principal adjusted for inflation will sink to about $\$ 86$ over the five-year term of the bond (Bouchet, 2004).

\section{Conclusion \& Policy Implication}

Study investigates the relationship between domestic terrorism per 100,000 population and investment/GDP among Muslim and Christian developing countries from 1990-2015. The robustness and consistency of estimates is ensured by applying different econometric techniques with different specification of models. The study applies ordinary least square (OLS) to ensure the presence of relationship. Heteroskedasticity in model leads to the application of Feasible Generalized Least Square (FGLS). Results of FGLS reveal that increase in incidence of domestic terrorism will decrease the level of investment among both groups of developing countries. However, economic growth improves the level of investment. The study also applies System Generalized Method of Moment (SGMM) due to presence of endogeneity. A standard deviation increase in terrorism per 100,000 populations will decrease the level of investment greater among Muslim developing countries as compared to Christian developing countries. The severity of terrorism is greater among Muslim developing countries as compared to Christians developing economies. Keeping in view all three possible techniques of OLS, FGLS and SGMM, it is seen that GDP growth improves the level of investment. 
Furthermore, military expenditure exhibits positive and significant relationship with investment among Muslim and Christian developing countries in all specification. Security of any country is an important indication for the involvement of foreign and private investor to make investment. Increase in military expenditure makes country more secure from terrorism and other conflicts, which will increase the level of investment in a country. In addition, majority of the other controlled variables such as economic growth, trade openness, education, exchange rate and inflation shows positive and significant impact on investment. However, internal and external conflicts show different results with different specification of model. There is a need to ensure the security of Muslim developing countries in order to increase the investment among all developing countries. However, policy makers of Muslim countries should focus on both types of terrorism in order to increase the level of investment. Our findings suggest that macroeconomic consequences of terrorism are quite significant. It confirms the prerequisite at part of public policy to mitigate the associated risk at best possible level.

\section{References}

Abadie, A., \& Gardeazabal, J. (2003). The Economic Cost of Conflict: A Case Study of the Basque Country. American Economic Review, 93, 113-32.

Abadie, A., \& Gardeazabal, J. (2008). Terrorism and World Economy. European Economic Review, 52, 127.

Adler, N. (2006). Corporate Global Citizenship: Successfully Partnering with the world. Corporate Strategies Under International Terrorism and Adversity, 177-95.

Arshed, N., ur Rehman, F., \& Aziz, O. (2019). An investigation into entrepreneurship determining factors The case of Asian economies. Organization Theory Review, 3(1), 1-20.

Asiedu, E. (2002). On Determinants of Foreign Direct Investment to Developing Countries: Is Africa Different? World Development, 78, 268-75.

Bandyopadhyay, S., Sandler, T., \& Younas, J. (2014). Foreign Direct Investment, aid and Terrorism. Oxford Economic Papers, 25, 25-50.

Becker, G., \& Murphy, K. (2001). Prosperity will rise out of the ashes. Wall Street Journal, 29, A22

Blomberg, S., \& Hess, G. (2008). From (no) Butter to Guns? Understanding the Economic Role in Transitional Terrorism. Cambridge University Press, 83-115.

Blomberg, S., Hess, G., \& Orphanides, A. (2004). The Macroeconomic Consequences of Terrorism. Journal of Monetary Economics, 51, 1007-32.

Bouchet, M. (2004). The Impact of Geopolitical Turmoil on Country Risk and Global Investment Strategy. The Security Business Nexus, 83-104.

Busse, M., \& Hefeker, C. (2007). Political risk, institution and foreign direct investment. European journal of political economy, 23, 397-415.

Blonigen, B. (2005). A review of the empirical literature on FDI determinants. Atlantic Economic Journal, $33,383-403$.

Choi, S. (2014). Causes of Domestic Terrorism. Koran Journal of International Studies, 12, 137-159.

Czinkota, M., Knight, G., \& Liesch, P. (2004). Terrorism and International Business: Conceptual Foundation. In G. Suder (Ed.), Terrorism and the International Business Environment. The Security Business Nexus, 43-57.

Deger, S., \& Sen, S. (1983). Military Expenditure: Spin-off and Economic Development. Journal of Development Economics, 13(1-2), 67-83.

Enders, W., \& Hoover, G. (2012). The Nonlinear Relationship Between Terrorism and Poverty. The American Economic Review, 102, 267-72.

Enders, W., Sachsida, A., \& Sandler, T. (2006). The Impact of Transitional Terrorism on US Foreign Direct Investment. Political Research Quarterly, (59), 517-31.

GCRI. (2015). Global conflict risk index. accessed from http://conflictrisk.jrc.ec.europa.eu/.

Glick, R., \& Rose, A. (2002). Does Currency Union affects trade? A Time series Evidence. European Economic Review, 46, 1125-51. 
Global Peace Index. (2015). Measuring Peace, its Causes and its Economic Values. Institue of Economic and Peace.

Global Report. (2009). Integrated network for societal conflict research, Center for Systemic Peace, Vienna: data available at http://www.systemicpeace.org/.

GTD. (2015). Global Terrorism Database . accessed from https://www.start.umd.edu/gtd/.

Innes, M. (2007). Denial of Sanctuary: Understanding Terrorist safe Havens. Westport, CN: Prager.

Kalim, R., Faiz, I., \& Arshed, N. (2019). Investor Confidence and Asymmetric Effects of Terrorism - A case of Pakistan, Transition studies review, 26(2).

Keefer, P., \& Loayza, N. (2008). Terrorism, Economic Development and Political Openness. Cambridge University Press. New York.

Kunreuther, H., Micheal-Kerjan, E., \& Baverly, P. (2003). Assessing managing and financing extreme events: Dealing with terrorism. Working paper 10179.

Li, Q., \& Schaub, D. (2004). Economic Globalization and Transitional Terrorism. Journal of Conflict Resolution, 48, 230-58.

Narayan, P.K., Narayan, S. \& Mishra, S. (2007) Do Remittances Induce Inflation? Fresh Evidence from Developing Countries. Southern Economic Journal, 77(4), 914-933.

Shahbaz, M. (2013). Linkages Between Inflation, Economic Growth and Terrorism in Pakistan. Economic Modelling, 32, 496-506.

Shahbaz, M., \& Shabbir, M. (2012). Military Spending and Economic Growth in Pakistan: New Evidence from Rolling Window Approach. Ekonomska Istrazivanja, 25(1), 144-159.

Spich, R., \& Grosse, R. (2005). How does Homeland Security affect US Firms, International Competitivenss? Journal of International Management, 11, 457-78.

Wagner, D. (2006). The Impact of Terrorism on foreign Direct Investment. International risk Management Institue.

WDI. (2015). World Development Indicators. World Bank. 